\title{
Coverage of Exposed Lower Extremity Bypass Vascular Graft with Integra and NPWT for Limb Salvage
}

\author{
Abou Issa ${ }^{1}$, Simman $\mathrm{R}^{*_{1,2}}$ and Gilkeson $\mathrm{JM}^{3}$ \\ ${ }^{1}$ Department of Pharmacology \& Toxicology, Boonshoft School of Medicine, Wright State University, USA \\ ${ }^{2}$ Plastic and Reconstructive Surgery, Boonshoft School of Medicine, Wright State University, USA \\ ${ }^{3}$ Department of Surgery, Section of Vascular and Endovascular Surgery, Boonshoft School of Medicine, Wright State \\ University, USA
}

${ }^{*}$ Corresponding author: Simman R, Wright State University Boonshoft School of Medicine, Wright State University, USA, E-mail: plasticsimman@yahoo.com

Citation: Abou Issa, Simman R, Gilkeson JM (2015) Coverage of Exposed Lower Extremity Bypass

Vascular Graft with Integra and NPWT for Limb Salvage. J Case Rep Stud 3(4): 401. doi: 10.15744/2348-

9820.3.401

Received Date: April 28, 2015 Accepted Date: August 07, 2015 Published Date: August 10, 2015

\begin{abstract}
Integra was first used to replace the dermis in third-degree burns to minimize contraction and scarring, its usage had led to better functional and aesthetic results. It acts as a network for dermal reconstruction. Its usage had expanded in trauma and soft tissue defects resulting after cancer excision and other defects with tendons and bone exposure. Integra has unlimited supply compared with skin grafts and flaps and it avoids donor site morbidity. This case study shows the promising use of Integra when applied over an exposed vascular graft in the lower extremity for limb salvage.
\end{abstract}

Keywords: Integra; Exposed bypass graft; NPWT

\section{Introduction}

Integra $^{\mathrm{Tm}}$ is a bi-laminar dermal regeneration template which was developed to treat acute large burns and is applied as a primary coverage. Nowadays, use of Integra is expanded to include coverage of soft tissue defects [1,2]. It acts as a network for dermal reconstruction. The first layer is composed of cross-linked bovine collagen and shark chondroitin-6-sulphate. The other layer, which is mainly an epidermal layer, consists of a thin layer of silicone [3,4]. The deeper layer acts as a "scaffold" for the host fibroblast to migrate, proliferate, and produce the collagen and new dermis $[4,5]$.

Integra was approved by the U.S Food and Drug Administration for use in acute burns in 1996 [2]. The advantage of Integra is to reduce morbidity of the donor site. Minimizing scar formation had made Integra useful as an adjunct dressing for the management of soft tissue defects and post burn scars [6]. This extended the usage of Integra to include both traumatic wounds and other reconstructive procedures [2]. In our case Integra was used to cover an exposed lower extremity vascular graft. The patient has given his consent for publishing his case.

\section{Case Report}

The patient is 56-year-old male with a history of diabetes mellitus (DM) type II, Peripheral Vascular Disease (PVD), Coronary Artery Disease and Chronic Obstructive Pulmonary Disease. He underwent five previous bypass surgeries, three on his right leg and two on his left leg, status post left below knee amputation. The summery of the right leg includes a femoral above knee popliteal bypass with reversed greater saphenous vein for critical limb ischemia that resolved the wound over several months. Two years later, this bypass failed and a second wound developed. Patient did have ultrasound surveillance once in two years that demonstrated graft patency. Patient's second wound had a Society for Vascular Surgery Lower Extremity Threatened Limb (SVS WIfI) classification of WIfI 232. Thrombolysis was attempted with EKOS and tPA unsuccessfully over 3 days. The patient had no available arm or leg veins. Cryopreserved vein was not an option secondary to patient height and length of occlusion needing greater than $80 \mathrm{~cm}$ of leg bypass. A second leg profunda femoris to posterior tibial bypass was created using ringed ePTFE (Propaten). This became infected at the groin and was explanted in its entirety. Four weeks after explantation and clinical resolution of infection, a third bypass with ringed ePTFE (Propaten) from right external iliac to a vein patched distal posterior tibial bypass was created to salvage his limb. This lower leg wound developed a surgical site infection leaving his synthetic bypass graft exposed on the medial aspect of his right leg (Figure 1).

The patient was referred to plastic surgery by his vascular surgeon in an attempt to salvage his foot and cover the exposed bypass graft to maintain patency. Under general anesthesia, the wound bed was carefully debrided using a curette, copious irrigation was made using antibiotics solution. After obtaining homeostasis the Integra was applied over the wound bed to cover the exposed graft and was sutured in place using multiple 4-0 chromic catgut. A Xeroform gauze was used to cover the Integra, then negative 
pressure wound therapy (NPWT) VAC dressing was applied for a total of three weeks and was set up at $75 \mathrm{mmHg}$ continuous pressure.

The patient was discharged on oral Doxycycline, Aspirin and Coumadin and was followed weekly at the wound center. Figure 2 shows the Integra covering the graft one week later. Figure 3, shows the graft at 3 months post operatively with patent bypass graft. The graft remained patent at 6 months but on ultrasound surveillance demonstrated it needed a distal anastomosis angioplasty as assisted primary patency. At one year post op, the graft thrombosed and $72 \mathrm{hrs}$ of open thrombectomy and outflow endovascular thrombolysis to preserve his last option was unsuccessful. The patient presented with gangrenous right foot which required right below knee amputation with total PTFE graft explantation.

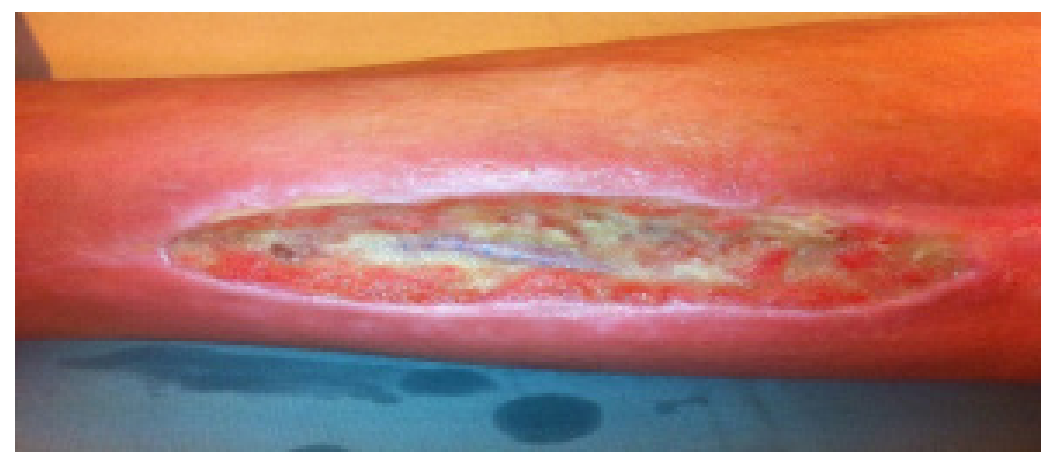

Figure 1: Shows the medial aspect of the right leg with exposed synthetic bypass graft

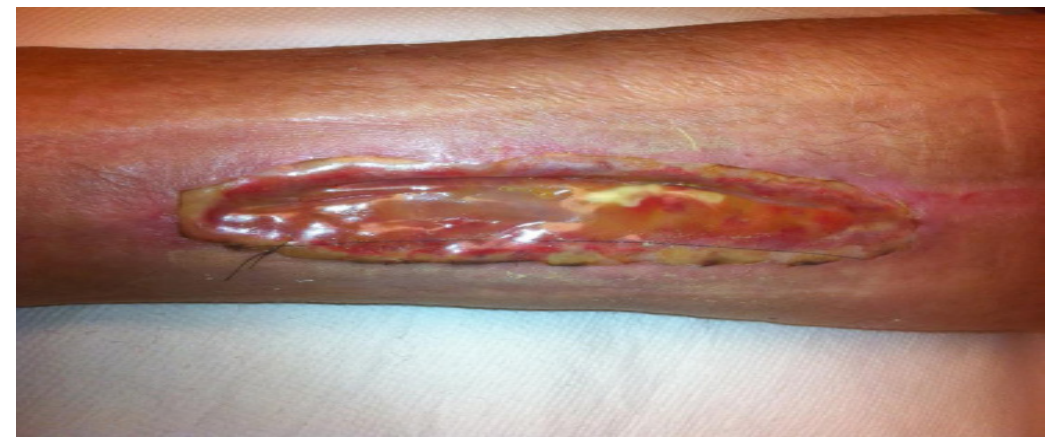

Figure 2: Shows the Integra covering the graft one week post operatively

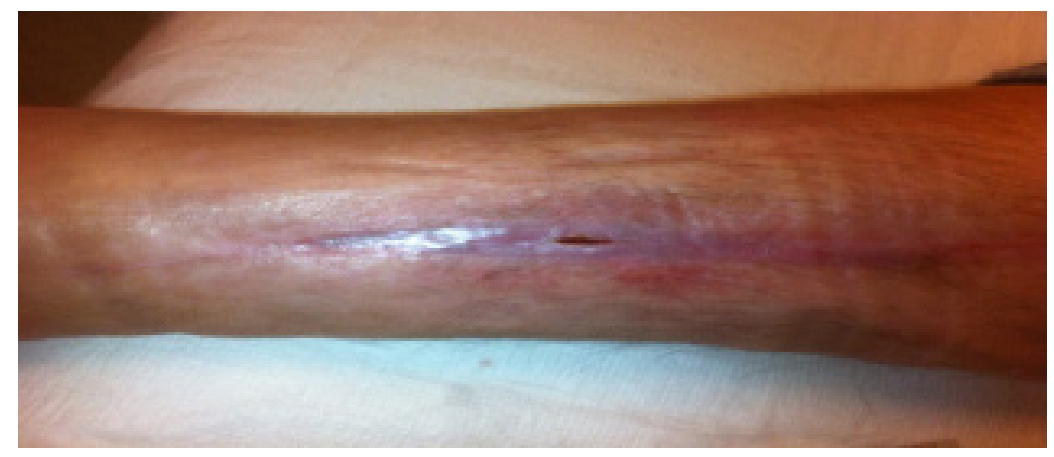

Figure 3: Shows the graft 3 months post operatively with patent bypass graft

\section{Discussion}

Wounds with exposed vessels, especially in artery bypass procedures, can pose a barrier to adequate skin healing. Skin grafts or flaps are sometimes difficult to perform in the face of critical limb ischemia. We report a case of a 56-year-old man who presented with necessitating salvage of the lower limb using artery bypass surgery. Immediate exposure of the lower leg ePTFE secondary to skin necrosis following the bypass led us to propose an innovative mean of wound coverage using Integra ${ }^{\circledR}$, a well-known dermal regeneration template.

The wound healed uneventfully with an appearance similar to that of the adjacent skin. Integra ${ }^{\bowtie}$ seems to be less demanding in terms of the vascular wound bed and the degree of oxygenation than a conventional skin graft. This finding could support further indications for this dermal regeneration template.

This case study shows the promising effect of Integra when applied over an exposed vascular graft. PVD makes wound healing very challenging because ischemia itself hinders the wound healing [7]. Applying skin graft over the bypass graft wasn't going to be 
successful and durable. The patient wasn't a good candidate for pedicle or free flap coverage due to severe PVD. Unlike skin grafts, Integra acts as a bridge by which revascularization starts from the terminal part of the Integra and gradually spreads out from the edges and covers a non-vascularized lower layer. This condition is known as bridging phenomenon. This was also supported by another animal study in which exposed tendons were covered with Integra $[8,9]$. In contrast to skin graft, Integra will less depend on the vascularity of the wound bed. Therefore, it is worth considering Integra as a good skin substitute especially for patients suffering from ischemia and peripheral vascular disease like in this case.

Other advantage of using Integra is the unlimited supply compared with skin grafts and flaps. Also, donor site morbidity is minimized or avoided. The other important benefit of applying Integra is that the skin will regenerate and regain its shape and function [2,7]. However, the risk of infection and the cost effectiveness are considered major disadvantages of Integra [3]. One may raise the question of why an alternative site vein or cryopreserved vein graft was not used in this case. Our patient already had right arm cephalic vein harvest for previous left leg bypass. Lesser Saphenous and left arm veins were too small for bypass. Cryopreserved saphenous vein was not an option given the fact that the distance from the external iliac artery to distal posterior tibial vessel was $92 \mathrm{~cm}$ and that the maximum length of cryopreserved vein is $80 \mathrm{~cm}$. Given these restrictions $100 \mathrm{~cm}$ PTFE with vein patch was the only long leg limb salvage option.

\section{Conclusion}

By the use of Integra and NPWT we were able to achieve limb salvage in this case for a year in a patient with otherwise threatened limb with exposed bypass graft and no other options are avalable.

\section{References}

1. Giovannini U, Teot L (2009) The use of Integra dermal regeneration template in treating Hidradenitis suppurativa - A clinical evaluation. J Plast Reconstr Aesthet Surg 62: 830S-1S.

2. Bache SE, Watson SB (2011) Bedside application of integra after debridement of necrotising fasciitis. J Plast Reconstr Aesthet Surg 64: 559-60.

3. Pollard RL, Kennedy PJ, Maitz PK (2008) The use of artificial dermis (Integra) and topical negative pressure to achieve limb salvage following soft-tissue loss caused by meningococcal septicaemia. J Plast Reconstr Aesthet Surg 61: 319-22.

4. Lohana P, Hassan S, Watson SB (2014) Integra ${ }^{\mathrm{TM}}$ in burns reconstruction: Our experience and report of an unusual immunological reaction. Ann Burns Fire Disasters 27: 17-21.

5. Moiemen NS, Staiano JJ, Ojeh NO, Thway Y, Frame JD (2001) Reconstructive surgery with a dermal regeneration template: clinical and histologic study. Plast Reconstr Surg 108: 93-103.

6. Stiefel D, Schiestl C, Meuli M (2010) Integra Artificial Skin for burn scar revision in adolescents and children. Burns 36: 114-20.

7. Michot A, Chaput B, Gobel F, Menez T, de Bonnecaze G, et al. (2015) Chronic ischaemia does not appear to hinder healing with Integra ${ }^{\circledR}$ implementation at a tibial artery bypass site. Int Wound J doi: 10.1111/iwj.12408.

8. Duteille F, Perrot P, Pannier M, Hubert L (2004) Evaluation of the advantages of Integra for covering Chinese flap graft donor sites: a series of 10 cases. Plast Reconstr Surg 114: 264-6.

9. Hulsen J, Diederich R, Neumeister MW, Bueno RA Jr (2014) Integra dermal regenerative template application on exposed tendon. Hand (N Y) 9: 539-42.

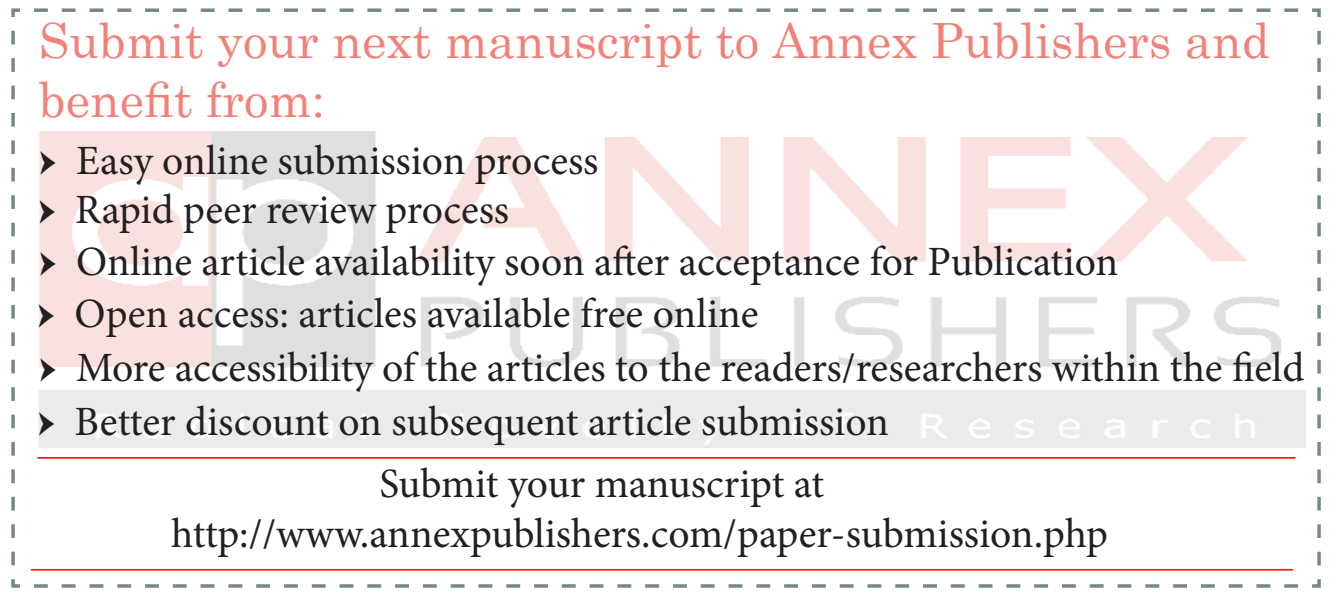

